\title{
ON NONVANISHING OF $L$-FUNCTIONS
}

\author{
BY FREYDOON SHAHIDI 1
}

The nonvanishing of Hecke $L$-functions at the line $\operatorname{Re}(s)=1$ has proved to be useful in the theory of uniform distribution of primes. One of the generalizations of this fact is due to H. Jacquet and J. A. Shalika [4], who proved the nonvanishing of the $L$-functions considered in [2]. The following theorem generalizes this result to the $L$-functions attached to the pairs of cusp forms on $G L_{n} \times G L_{m}$ (cf. [3]). It appears to have an application in the classification of automorphic forms on $G L_{n}$ (communications with H. Jacquet and J. A. Shalika).

Let $F$ be a number field and denote by $\mathbf{A}$ its ring of adeles. Fix two positive integers $m$ and $n$. Let $\pi$ and $\pi^{\prime}$ be two cuspidal representations of $G L_{n}$ (A) and $G L_{m}(\mathrm{~A})$. Fix a complex number $s$. Write $\pi=\bigotimes_{v} \pi_{v}$ and $\pi^{\prime}=\bigotimes_{v} \pi_{v}^{\prime}$, where $\pi_{v}$ and $\pi_{v}^{\prime}$ denote the $v$ th components of $\pi$ and $\pi^{\prime}$ at each place $v$ of $F$, respectively. Let $S$ be the finite set of all ramified places, including the infinite ones. For every finite place $v$, H. Jacquet, I. I. Piatetski-Shapiro, and J. A. Shalika have defined (cf. [3]) a local $L$-function $L\left(s, \pi_{v} \times \pi_{v}^{\prime}\right)$. Let

$$
L_{S}\left(s, \pi \times \pi^{\prime}\right)=\prod_{v \notin S} L\left(s, \pi_{v} \times \pi_{v}^{\prime}\right) .
$$

Put $i=(-1)^{1 / 2}$. Then we have

Throrem. $L_{S}\left(1+i t, \pi \times \pi^{\prime}\right) \neq 0$ for $\forall t \in \mathbf{R}$.

OUTLINE OF THE PROOF. The proof follows the general principle of applying Eisenstein series to $L$-functions which is due to R. P. Langlands [5] (same as in [4]). Put $G=G L_{n+m}$ and $M=G L_{n} \times G L_{m}$. Consider $M$ as a Levi factor of a maximal standard parabolic subgroup of $G$. Choose $\varphi$ in the space of ${ }^{\circ} \pi=\tilde{\pi} \otimes \pi^{\prime}$, where $\tilde{\pi}$ denotes the contragredient of $\pi$. Extend $\varphi$ to $\tilde{\varphi}$, a function on $G(\mathbf{A})$, as in [7]. Put

$$
\Phi_{s}(g)=\delta_{p}^{s-1 / 2}(p) \widetilde{\varphi}(g)
$$

where $P=M N, g=k p, p \in P(\mathrm{~A})$, and $k \in K$. Here $K=\Pi_{v} K_{v}$ is a maximal compact subgroup of $G(\mathrm{~A})$ such that $K_{v}=G\left(O_{v}\right)$ for every finite $v$. Now set (cf. [6], [7])

Received by the editors November 7, 1979.

AMS (MOS) subject classifications (1970). Primary 12A70, 12B30; Secondary 10D40.

1 Partially supported by NSF grant MCS 79-02019. 


$$
E(s, \tilde{\varphi}, g, P)=\sum_{\gamma \in G(F) / P(F)} \Phi_{s}(g \gamma)
$$

the Eisenstein series attached to $\varphi$. Consider

$$
E_{\chi}(s, \tilde{\varphi}, g, P)=\int_{U(A) / U(F)} E(s, \tilde{\varphi}, g u, P) \overline{\chi(u)} d u,
$$

where $U$ is the subgroup of upper triangulars in $G$ with ones on diagonals, and $\chi$ is a nondegenerate character of $U(\mathrm{~A}) / U(F)$. Now for each place $v$, let

$$
\Pi_{v}=\underset{P\left(F_{v}\right) \uparrow G\left(F_{v}\right)}{\operatorname{Ind}}\left(\left({ }^{\circ} \pi_{v}\right)_{\infty} \otimes \delta_{P, v}^{s}\right)
$$

and denote by $\lambda_{v}$ the Whittaker functional attached to $\Pi_{v}$ as in [1], [7], and [8]. Put

$$
W_{s, v}(g)=\lambda_{v}\left(\Pi_{\nu}\left(g^{-1}\right) f_{s, v}\right) \quad\left(g \in G\left(F_{v}\right)\right)
$$

where $f_{s, v}$ is defined as in Lemma 4.1 of [7]. Then for $\operatorname{Re}(s)<-1 / 2$,

$$
E_{\chi}(s, \tilde{\varphi}, g, P)=\prod_{v} w_{s, v}\left(g_{v}\right) \quad\left(g=\left(g_{v}\right) \in G(\mathbf{A})\right)
$$

It is proved in [1] and [8] (also see [7]) that at every $v, W_{s, v}$ may be so chosen that $W_{s, v}(e) \neq 0$. Now write

$$
E_{\chi}(s, \tilde{\varphi}, e, P)=\prod_{v \in S} w_{s, v}(e) \cdot \prod_{v \notin S} w_{s, v}(e)
$$

Then by the previous remark, we may choose $\varphi$ such that $\Pi_{v \in S} W_{s, v}(e)$ is nonzero.

It is a result of W. Casselman and J. A. Shalika [1] that if $v$ is unramified, $\varphi$ can be so chosen that

$$
W_{s, v}(e)=L\left(-(n+m) s+1, \pi_{v} \times \pi_{v}^{\prime}\right)^{-1},
$$

and therefore

$$
L_{S}\left(-(n+m) s+1, \pi \times \pi^{\prime}\right)^{-1}=\prod_{v \notin S} W_{s, v}(e) .
$$

Now the theorem follows from the fact that $E(i t, \tilde{\varphi}, e, P)$ and consequently $E_{\chi}(i t, \tilde{\varphi}, e, P)$ are both holomorphic for all $t \in \mathbf{R}$ (cf. [6]).

\section{BIBLIOGRAPHY}

1. W. Casselman and J. A. Shalika, The unramified principal series of P-adic groups. II, The Whittaker functions (preprint).

2. R. Godement and H. Jacquet, Zeta functions of simple algebras, Lecture Notes in Math., vol. 260, Springer-Verlag, Berlin and New York, 1972.

3. H. Jacquet, From $G L(2)$ to $G L(n), 1975$, U.S.-Japan Seminar on Number Theory, Ann Arbor, Michigan. 
4. H. Jacquet and J. A. Shalika, A non-vanishing theorem for zeta functions of $G L_{n}$, Invent. Math. 38 (1976), 1-16.

5. R. P. Langlands, Euler products, Yale Univ. Press, New Haven, 1971.

6. - On the functional equation satisfied by Eisenstein series, Lecture Notes in Math., vol. 544, Springer-Verlag, Berlin and New York, 1976.

7. F. Shahidi, Functional equation satisfied by certain L-functions, Compositio Mathematica 37 (1978), 171-208.

8. _- Whittaker models for real groups, Duke Math. J. (to appear).

DEPARTMENT OF MATHEMATICS, PURDUE UNIVERSITY, WEST LAFAYETTE, INDIANA 47907 